\title{
ÉLEVAGE DU SAUMON ATLANTIQUE (S. SALAR) EN ÉCLOSERIE : ESSAI D'UTILISATION DE SUBSTRATS DURANT LA RÉSORPTION VITELLINE
}

\author{
J.L. GAIGNON, P. PROUZET \\ Centre Océanologique de Bretagne - B.P. 337 - 29273 BREST Cédex
}

\begin{abstract}
L'expérience acquise par le Centre Océanologique de Bretagne en matière d'alevinage de juvéniles de saumon atlantique a montré que les difficultés essentielles résidaient dans la phase de démarrage (de l'éclosion au ler été). Ainsi une précédente expérimentation (J.L. GAIGNON et P. PROUZET, 1981) a mis en évidence que les conditions d'incubation durant la phase de résorption pouvaient avoir des conséquences très néfastes avec perte de poids considérable de l'alevin au moment de la prise d'alimentation. Or, plus les alevins sont gros plus le "démarrage " est aisé (L.A. LEON et W.A. BONNEY, 1979): Le présent essai a pour but de remplacer les fonds d'auges lisses utilisés actuellement qui demandent à l'alevin un effort de nage soutenu et permanent pour se maintenir en place, ce qui entraîne des besoins importants en énergie utilisée aux dépens de la croissance. Nous essaierons donc de trouver un substrat rugueux pouvant favoriser un certain soutien de l'alevin tout en le soustrayant partiellement à l'action du courant, afin d'obtenir des conditions plus proches des conditions naturelles sans pour autant utiliser des lits de gravier (Anonyme, 1975; DUMAS, 1978) dont l'emploi est peu commode. Un tel système permettrait, sans diminuer les taux de renouvellement de l'eau, de créer un environnement plus favorable au développement de l'alevin jusqu'à la prise d'alimentation.
\end{abstract}

Un tel substrat doit de plus être simple et peu onéreux, être utilisable dans les auges d'alevinage traditionnelles, afin d'éviter le recours à des systèmes d'incubation beaucoup plus sophistiqués type "Heath" (ou "Heath" amélioré: K.A. LEON and W.A.BONNEY, 1979), "Auke bay" (F.M.SALTER, 1975), ou jarres d'incubation (K.A. LEON, 1975).

A cet effet, divers systèmes fabriqués artisanalement pour l'expérience ont été testés ainsi qu'une moquette industrielle, matériau voisin de l' "Astroturf" ou tapis brosse en plastique couramment utilisé pour l'alevinage des salmonidés sur la côte Pacifique (BAYLEY and TAYLOR, 1974).

\section{I - MATÉ RIEL et méthOdes}

\subsection{Structures et techniques d'élevage, matériel biologique}

Les structures d'expérimentation sont des auges en plastique noir $(180 \times 30 \mathrm{~cm}$ utile, hauteur d'eau $12-15 \mathrm{~cm}$ ) pourvues de couvercles. Les débits sont maintenus à $8 \mathrm{l} / \mathrm{mn}$ en moyenne $(6 \mathrm{a} 10 \mathrm{l} / \mathrm{mn})$ : débit minimum pour assurer l'élimination des déjections. Les œufs sónt incubés sur des clayettes en bois à fond constitué d'un filet à plancton.

Les auges sont alimentées à partir d'un ruisseau dont l'eau est de qualité favorable a l'élevage des salmonidés. La température de l'eau durant l'essai est de $9,4^{\circ} \mathrm{C}$ en moyenne (minimum : $7,5^{\circ} \mathrm{C}$, maximum : $11,0^{\circ} \mathrm{C}$ ). Par contre, les fortes précipitations du mois de mars 1981 ont considérablement augmenté la turbidité et entraîné des dépôts (terre et débris végétaux) pouvant atteindre $1 \mathrm{~cm}$ d'épaisseur au fond des auges.

Les œufs utilisés proviennent de géniteurs capturés sur un ruisseau pépinière du bassin de l'Elorn et issus d'une opération de repeuplement extensif. L'expérience a débuté le 4 février 1981. Deux lots d'œufs I et Il (obtenus par mélange de plusieurs pontes) d'un poids moyen de $144,8 \mathrm{mg}$ et $139,0 \mathrm{mg}$ respectivement, ont été utilisés. La mortalité de chaque lot avant l'essai est de $5,1 \%$ et $16,3 \%$ respectivement. La mortalité plus importante du lot ll est due aux mauvaises conditions d'incubation d 'une des pontes, la mortalité moyenne des autres pontes du lot étant de 6,9\%. 
Tous les œufs ont éclos le 9-12 février. Après l'éclosion, les alevins gagnent seuls - sans aucune manipulation - le fond de l'auge sur lequel a été disposé le substrat à tester.

L'essai se termine le 24 mars, date de la prise d'alimentation.

\subsection{Description des différents substrats}

Les différents substrats sont installés lors du début de l'expérience, donc avant l'éclosion, et retirés avant la prise d'alimentation.

Les substrats utilisés sont les suivants:

\section{Lot I}

- Fond lisse d'une auge (témoin)

- Moquettes industrielles de type "tapis de propreté Nomad" (1) $100 \%$ vinylique, l'une à petite boucle (sans thibaude), l'autre à grosse boucle (cf photo 1).

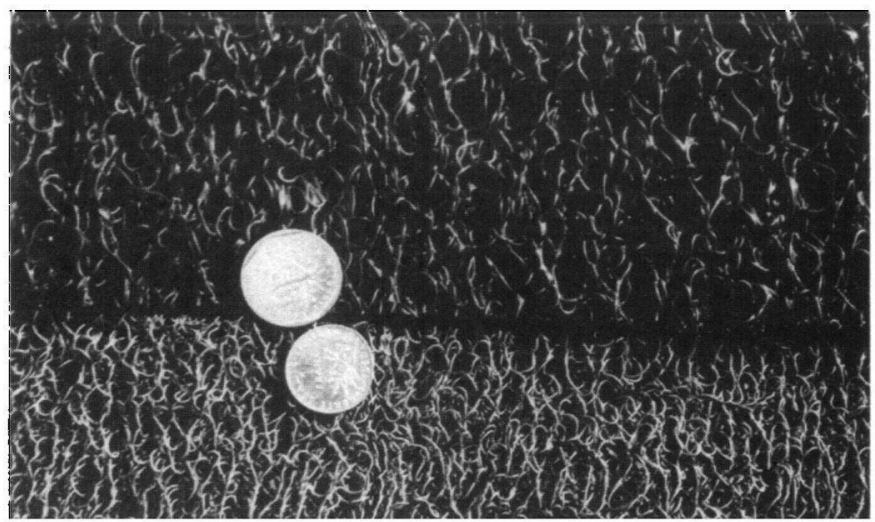

Photo 1: Moquettes industrielles testées dans l'expérience : en haut, modèle "petite boucle" ; en bas, modèle " grosse boucle".

Lot II

- Fond lisse d'une auge (témoin)

- Grille métallique galvanisée, type "caillebotis", épaisseur $25 \mathrm{~mm}$, taille des mailles: $30 \times 30 \mathrm{~mm}$; (cf photo 2).

- Cadre en bois sur lequel on tend sur les deux faces un grillage en plastique type "Netlon", épaisseur du cadre : $25 \mathrm{~mm}$, taille des mailles : $10 \mathrm{~mm}$; (cf photo 2).

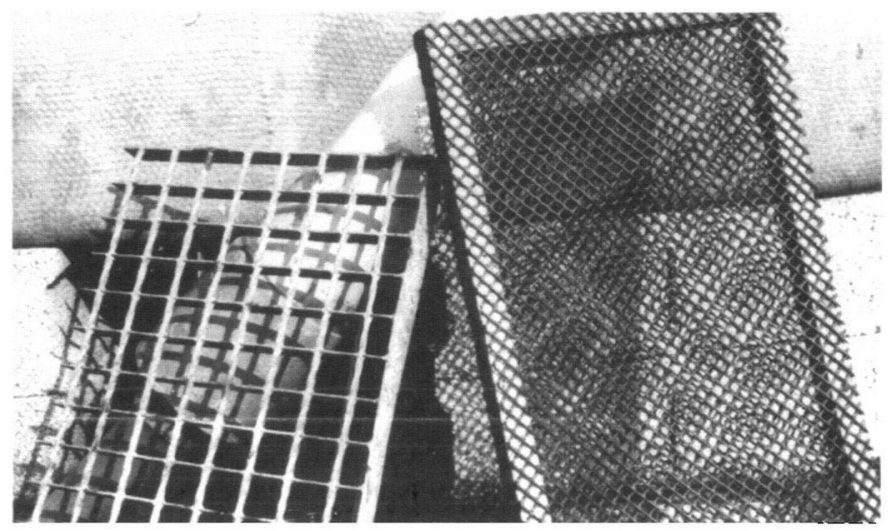

Photo 2: Substrats de fonds d'auge : à droite, cadre en bois tendu de grillage plastique; à gauche, grille métallique.

- Les deux substrats précédents reposent entièrement sur le fond de l'auge et sont par conséquent totalement inamovibles tant que les alevins ne nagent

(1) Échantillons fournis par 3 M France - 10 rue Voltaire 44000 NANTES. 
pas. Afin de faciliter les interventions (nettoyage, enregistrement hebdomadaire de la mortalíté), des substrats identiques ont été également utilisés én y ajoutant des pieds de $5 \mathrm{~mm}$ de haut qui permettent leur remise en place après interventions (cf photo 3 ).

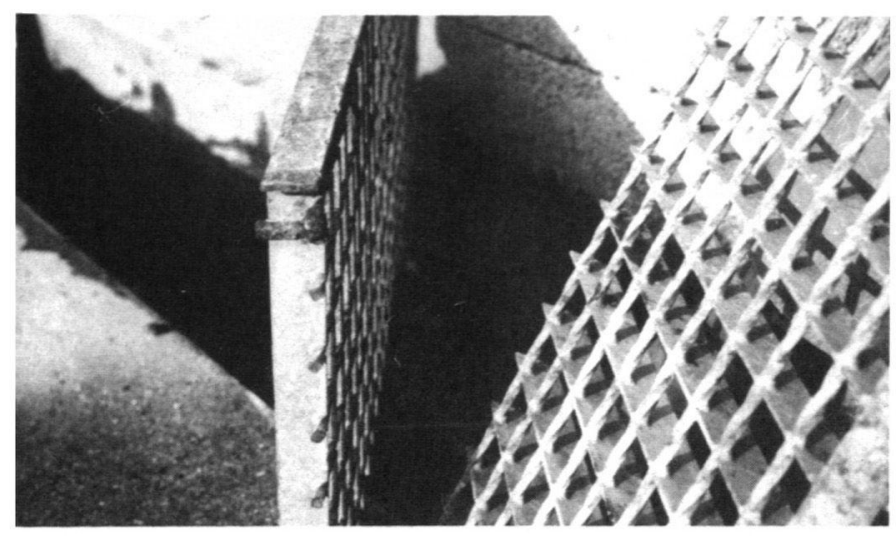

Photo 3: Substrat "“ pieds": à gauche, pied de $5 \mathrm{~mm}$ de haut sur grille métallique.

\subsection{Recueil des données}

Une visite de chaque bac est effectuée quotidiennement. Elle permet de dénombrer précisément et d'éliminer les morts des auges témoins, d'éliminer également les morts dérivés à la grille pour les autres auges. Pour les auges à substrat, la mortalité est relevée précisément, soit avec une fréquence hebdomadaire dans le cas des substrats "à pieds", soit globalement en fin d'expérience pour les substrats de fond, et ceci généralement en dénombrant les vésicules coagulées. Cette différence obligatoire de méthode de comptabilisation des morts est susceptible d'entraîner une sousestimation de la mortalité dans le cas des auges à substrat de fond.

Les gains de poids sont établis à partir des poids moyens (moyenne des pesées individuelles réalisées avec une balance de précision au $\mathrm{mg}$ ).

\section{II - RÉsultats}

- Les mortalités enregistrées figurent dans le tableau 1.

En ce qui concerne le lot $I$, la mortalité la plus faible a été enregistrée dans le cas de la moquette "petite boucle". Bien que non significativement différente de celle du témoin, elle est nettement inférieure $\left(X^{2}=9,5 ; p=0,005\right)$ à celle de l'auge à moquette "grosse boucle" qui a la mortalité la plus élevée. Dans ce cas, il semble que les alevins soient restés "coincés" dans les mailles puis se soient trouvés pris dans les dépôts décantés où ils se sont asphyxiés, ou bien se sont faufilés entre la moquette et le fond de l'auge sans pouvoir en sortir et ceci dès le début de la résorption au moment où les vésicules sont les plus volumineuses.

En ce qui concerne le lot II, les mortalités obtenues, quel que soit le substrat, sont voisines et légèrement inférieures à celles du témoin.

Les mortalités supérieures enregistrées sur le lot II par rapport au lot I sont dues aux mauvaises conditions d'incubation (turbulence prononcée dans une clayette) d'une des pontes mélangées pour obtenir le lot II.

- Les gains de poids obtenus par rapport au poids initial des œufs et par rapport aux témoins sont donnés dans le tableau 1.

Alors que les alevins élevés dans les deux auges-témoins à fond lisse ne présentent pas d'augmentation de poids, voire même une faible perte de poids dans le cas du lot $\mathrm{I}$, tous les autres lots présentent un gain de poids sensible. Pour le lot $\mathrm{I}$, le meilleur résultat est obtenu avec la " moquette grosse boucle" (accroissement pondéral de $25 \%$ ). Pour le lot II, les alevins sur substrats de fond ont des gains de poids du même ordre (25\%), alors que ceux élevés dans l'auge avec une grille "à pieds " ont un gain de poids intermédiaire $(10 \%$ environ). 


\begin{tabular}{|c|c|c|}
\hline 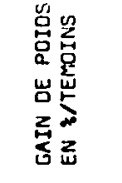 & $\begin{array}{ll}n & 0 \\
& \dot{m} \\
\dot{m} & +\end{array}$ & 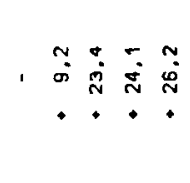 \\
\hline 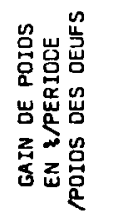 & $\hat{f} \stackrel{0}{=} \hat{n}$ & 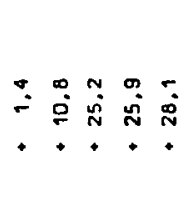 \\
\hline 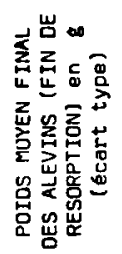 & 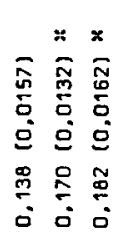 & 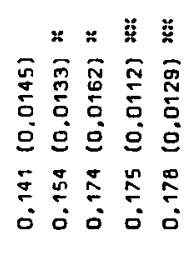 \\
\hline 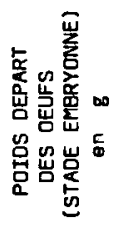 & $\frac{\hat{\theta}}{0} \frac{n}{0}$ & 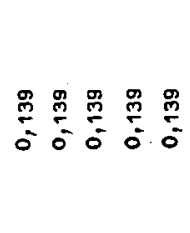 \\
\hline 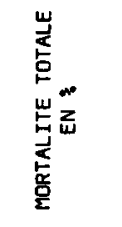 & 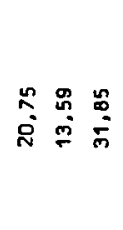 & 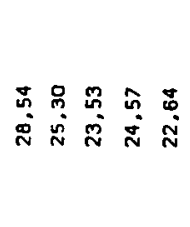 \\
\hline 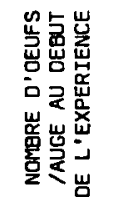 & 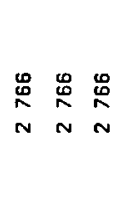 & 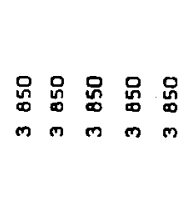 \\
\hline 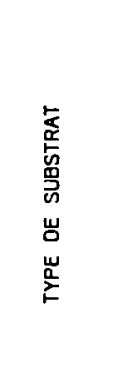 & 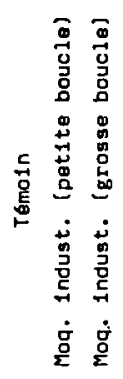 & 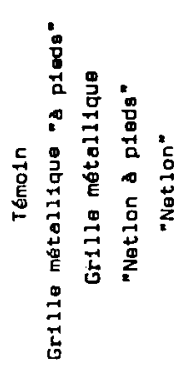 \\
\hline 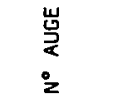 & $\sigma \pm$ & 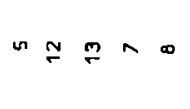 \\
\hline 5 & $H$ & $\ddot{H}$ \\
\hline
\end{tabular}

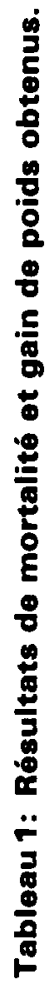




\section{III - DIsCUSSION - CONCLUSION}

Les différents substrats utilisés n'ont pas fait apparaître de différences significatives de mortalité par rapport aux lots élevés sur fond lisse selon la technique traditionnelle, en dépit de l'absence d'intervention de nettoyage (enlèvement des morts) et du manque de propreté apparent des auges. On remarquera en effet que la quantité de matière en suspension sur les auges "non entretenues" conduit à un colmatage important des substrats sans conséquences néfastes sur la survie des alevins, sauf dans le cas de la moquette "grosse boucle" pour laquelle les alevins s'extirpent difficilement de la boue. Dans tous les autres cas, on remarque que les alevins " nettoient ", grâce à leurs mouvements, la logette ou l'emplacement qu'ils occupent, ce qui leur permet de se trouver sur un support solide et propre. Il est possible en fait que l'absence d'intervention a priori préjudiciable aux conditions d'hygiène de l'élevage ait pu limiter le stress dû aux manipulations.

En ce qui concerne le gain de poids, on peut noter l'influence favorable de tous les substrats par rapport aux auges à fond lisse, ce qui confirme les résultats de K.A. LEON et W.A. BONNEY (1979) obtenus dans d'autres types de structures d'alevinage. Cependant, les substrats de fond ont donné des résultats supérieurs au substrat " métallique à pieds". Le grillage du substrat "netlon à pieds". légèrement détendu, reposait sur les dépôts décantés dans le fond de l'auge : son rôle et ses résultats sont identiques à ceux du substrat " netion sans pieds". Dans le cas du substrat "à pieds" , la vitesse du courant est probablement diminuée, mais le fond reste lisse sans possibilité de repos complet pour les alevins qui doivent toujours se maintenir en nageant. Plus que d'une diminution de la vitesse du courant, les alevins semblent donc avoir besoin d'un support complet que seul leur apporte un substrat très rugueux constituant en quelque sorte des logettes individuelles, ce qui confirme les expériences de MARR (1967).

Le meilleur gain de poids et les plus grosses mortalités sont obtenus avec la moquette "grosse boucle" ; cependant, il ne semble pas que l'on puisse faire de relation entre les deux. Par contre, les mailles de ce substrat ont une taille telle - très proche de la taille de la vésicule de l'alevin - qu'elles pourraient permettre un meilleur support et pourraient donc expliquer un meilleur gain de poids.

En conclusion, on retiendra pratiquement que l'on obtient un gain de poids de l'alevin appréciable (25 a 30\%) durant la phase de résorption avec tout substrat rugueux qui peut servir de " support " aux alevins. Le meilleur substrat essayé en ce qui concerne le gain de poids est la " moquette industrielle grosse boucle" avec laquelle on obtient cependant les plus grosses mortalités. La "moquette petite boucle " étant onéreuse, les grilles métalliques ou cadres tendus de grillage restent les meilleures solutions artisanales peu coûteuses ( $40 \mathrm{~F} / 3$ cadres tendus de grillage/auge, amortissable sur plusieurs années).

\section{BIBLIOGRAPHIE}

ANONYME. Annual Report noXX: 1975. Salmon Research Trust of Ireland.

BAYLEY J.E. and S.G. TAYLOR, 1974. Plastic Turf substitute for gravel in Salmon Incubators. Marine Fisheries Review n०1097: Vol. 36, n०10, p. 35-38.

DUMAS J., 1978. L'élevage intensif des jeunes saumons atlantiques de repeuplement : Bulletin scientifique et technique du département Hydroúiologie. INRA, n०4.

GAIGNON J.L. et P. PROUZET, 1981. Étude de la production de juvéniles de saumon atlantique (Salmo salar). If - Influence de la densité sur l'alevinage. Doc. Int. CNEXO, $35 \mathrm{p}$.

LEON K.A., 1975. Improved growth and survival of juvenile atlantic salmon (Salmo salar) hatched in drums packed with a labyrinthine plastic substrate. Prog. Fish. Cult. Vol. 37, no3, p. 158-163.

LEON K.A. and W.A. BONNEY, 1979. Atlantic Salmon Embryos and Fry: Effects of various Incubation and Rearing Methods on Hatchery, Survival and Growth. Prog. Fish. Cult. Vol. 41, no 1, p. 20-25.

MARR B.H.A., 1967. Factors affecting the growth of salmon alevins and their survival and growth during the fry stage. Ass. Riv. Auth. Year book, p. 133-141.

SALTER F.H., 1975. A new incubator for Salmonids designed by Alaska Laboratory. Marine Fisheries Review $n^{\circ} 1153$ : Vol. 37, n7, p. 26-29. 